\title{
Design and Construction of an Imaging beamline at the Nagoya University Neutron Source
}

\author{
${ }^{1}$ Nagoya University, Furo-cho, Chikusa, Nagoya, 464-8602, Japan \\ ${ }^{2}$ High Energy Accelerator Research Organization, Tokai, Ibaraki, 319-1106, Japan \\ ${ }^{3}$ RCNP, Osaka University, Mihogaoka, Ibaraki, Osaka, 567-0047, Japan \\ ${ }^{4}$ KyotoUniversity, Gokasho, Uji, Kyoto, 611-0011, Japan \\ ${ }^{5}$ KyotoUniversity, Kumatori, Osaka, 590-0494, Japan \\ ${ }^{6}$ Japan Atomic Energy Agency, Tokai, Ibaraki, 319-1106, Japan \\ ${ }^{7}$ RIKEN, Hirosawa, Wako, Saitama, 391-0198, Japan \\ ${ }^{8}$ Kyushu University, Motooka, Nishi, Fukuoka, 819-0395, Japan
}

Katsuya Hirota ${ }^{1, *}$, Shogo Awano ${ }^{1}$, Takuhiro Fujiie ${ }^{1}$, Seiso Fukumura ${ }^{1}$, Mayu Hishida ${ }^{1}$, Go Ichikawa $^{1,2}$, Sohei Imajo ${ }^{1,3}$, Ikuya Itoh $^{1}$, Yoshihisa Iwashita ${ }^{4}$, Masaaki Kitaguchi ${ }^{1}$, Yoshiaki Kiyanagi ${ }^{1}$, Yasutoshi Kuriyama ${ }^{5}$, Koki Morikawa ${ }^{1}$, Yudai Niinomi ${ }^{1}$, Hirohiko M. Shimizu ${ }^{1}$, Kazuki Tsuchida ${ }^{1}$, Yusuke Tsuchikwa ${ }^{1,6}$, Yukio Tsurita ${ }^{1}$, Akira Uritani ${ }^{1}$, Kenichi Watanabe $^{1}$, Yutaka Yamagata $^{7}$, Nana Yamamoto $^{1}$, Atsushi Yamazaki ${ }^{1}$, Sachiko Yoshihashi ${ }^{1}$, and Tamaki Yoshioka ${ }^{8}$

\begin{abstract}
The Nagoya University Accelerator driven Neutron Source (NUANS) is constructed at the main campus of the Nagoya University. The electrostatic accelerator is used with the maximum proton energy and intensity of $2.8 \mathrm{MeV}, 15 \mathrm{~mA}(42 \mathrm{~kW})$ respectively. Two neutron beamlines are designed at NUANS. The BL1 is dedicated to BNCT development. The BL2 is designed for research and development for neutron devices and neutron imaging. The neutrons used for the BL2 are generated by using the $(\mathrm{p}, \mathrm{n})$ reaction from a thin beryllium target. We constructed a compact target station for the BL2 and measured the neutron transmission image.
\end{abstract}

\section{Introduction}

Neutron is a very good probe to investigate the inner structure of materials. Large neutron facilities like J-PARC MLF and SNS have been constructed in this decade, and ESS facility is under construction. These large facilities are very good tools to study in the academic field. However, the opportunity to use them is very low, many neutron scientists use these facilities only once a year. The allocated beam time is usually shorter than 10 days on these powerful machines. The scientists require more neutron beam to study physics, chemistry, and engineering.

One of the solutions to solve this situation is to construct a Compact Accelerator-driven Neutron Source (CANS) [1]. The CANS has some merits compare to large neutron facilities. The constructing cost is lower than larger facilities. The opportunity to use the neutron beam is so higher. All devices from the accelerator to detectors is easy to handle, which is very good for student education. The amount of radiation is smaller than that of the large-scale facilities. Unfortunately, the emitted total neutron is much lower than large facilities. It is very important to consider what you can do with CANS and what you should do with large neutron facilities. We can use these CANS for BNCT(Boron Neutron Capture Therapy), neutron imaging, device development and so on.

*e-mail: hirota@phi.phys.nagoya-u.ac.jp
Nagoya University has constructed CANS using an electrostatic accelerator, Nagoya University Acceleratordriven Neutron Source (NUANS)[2-4]. Two neutron beamlines are designed at NUANS. The first beamline (BL1) is dedicated to BNCT development. The second beamline (BL2) is designed for research and development for neutron devices and neutron imaging. In this paper, we will present the current status of the NUANS BL2.

\section{NUANS}

Two beamlines are constructed at NUANS. In both beamlines, neutrons are provided by bombarding a high current proton beam to a lithium and beryllium target, respectively. The initial proton beam is provided by a Dynamitron electrostatic accelerator. The $2.8 \mathrm{MeV}$ proton beam used in NUANS has a maximum current of $15 \mathrm{~mA}$. The beam transport for each beamline is selected by using a dipole magnet located at $2.5 \mathrm{~m}$ downstream from the accelerator exit. The deflection angles from the initial beam axis to the beamlines are $20^{\circ}$ and $70^{\circ}$, respectively. After dividing the beamline, the proton beam hits the neutron generation target.

\section{Design and Construction of the BL2}

The BL2 is designed as a multipurpose beamline for radiography and device development. The neutron genera- 
tion target is installed $5 \mathrm{~m}$ downstream of the proton bending dipole magnet. The layout is shown in a previous paper $[2,3]$. Since this beamline uses a high-current proton beam, even if the center of the beam reaches the neutron generation target, in the case of the periphery hits the beam duct, the duct generates heat and eventually the duct breaks down. Therefore, it is necessary to monitor the beam loss so that the periphery of the beam does not hit the duct very much. A circular collimator with a diameter of $50 \mathrm{~mm}$ is installed just downstream of the bending magnet to prevent the tail of the proton beam that has passed through the bending magnet from hitting the beam duct. Also, a 4-quadrant slit is installed at a position of $3.8 \mathrm{~m}$ downstream of the bending magnet to limit the size of the proton beam irradiated to the neutron generation target, and by monitoring the proton current hitting each slit, to confirm the balance of the proton beam in vertical and horizontal directions. Both collimators are watercooled. A total of 16 thermocouple temperature gauges are attached to the proton beam duct, and the duct temperature is checked. If an abnormal temperature rise is detected, the accelerator beam is automatically stopped. In this way, the proton beam is controlled by inexpensive devices using the circular collimator, the 4-quadrant slit, and thermocouple gauges.

In this BL2, neutrons are generated using the $\mathrm{Be}(\mathrm{p}, \mathrm{n})$ reaction. Two types of target materials, beryllium and lithium, are candidates for neutron generation targets in this energy region [5]. Because beryllium target is chemically stable and easier to use than lithium, BL2 uses beryllium. The proton beam hit a $40 \mu \mathrm{m}$ thick beryllium foil. This foil is silver brazed to a $5 \mathrm{~mm}$ thick Vanadium plate. The thickness of the beryllium foil is designed so that the proton beam can penetrate the beryllium foil[6]. Cooling water flows on the back of this neutron generation target so that the heat generated by beam irradiation can be removed.

The radiation shield around the neutron generation target is using polyethylene blocks containing $10 \%$ of $\mathrm{B}_{2} \mathrm{O}_{3}$, and $\mathrm{Pb}$ blocks. Since the estimated maximum neutron energy is less than $1 \mathrm{MeV}$, it is not necessary to shield fast neutrons using iron plates. A multi-layer structure is adopted to shield radiation efficiently. A large amount of neutron flows from the neutron generation target to the upstream side of the proton beam duct, but the neutron shield cannot be inserted into the duct. So the proton beam duct is surrounded by polyethylene blocks containing $\mathrm{B}_{2} \mathrm{O}_{3}$.

A polyethylene block with a size of $100 \times 100 \times 100$ $\mathrm{mm}^{3}$ is used as a thermal neutron moderator. In general, water $\left(\mathrm{H}_{2} \mathrm{O}\right)$ or polyethylene is often used as the thermal neutron moderator in CANS $[1,7,8]$. The reason why polyethylene is used here is that the neutron intensity is not so high, the frequency of carbonization of polyethylene is not high, and it is easier not to use a container to improve the shape of the moderator in the future. Graphite blocks of the $50 \mathrm{~mm}$ thickness are used as a reflector around the moderator.

Neutrons emitted from the neutron moderator pass through the hole in the shield and are measured at the experimental port. There are two experimental ports at
BL2, BL2-short and BL2-long. The BL2-short and -long are on the same straight line. The length of flight path of the BL2-short is $50 \mathrm{~cm}$ and the BL2-long is $500 \mathrm{~cm}$. The corresponding $\mathrm{L} / \mathrm{D}$ values are 5 and 50 , respectively. In $\mathrm{L} / \mathrm{D}=5$, it is generally considered that measurement with sufficient quality is impossible when performing imaging measurement. However, if a measurement of sufficiently thin sample material is possible, measurement with highintensity neutrons is possible even in such a small neutron source. Currently it measured only at BL2-short, because of the low neutron beam intensity. The effective area of neutron beam at the BL2-short is $10 \times 10 \mathrm{~cm}^{2}$.

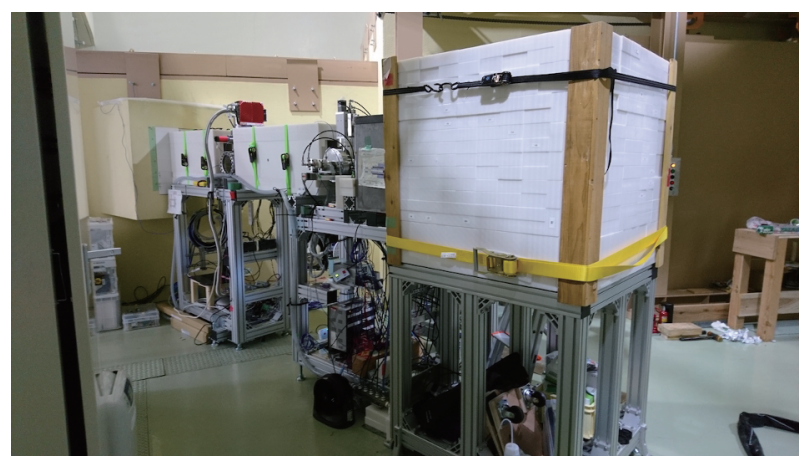

Figure 1. Photograph of NUANS BL2. Left hand side is the proton beam line. The center of the photo is the target shield.

Figure 1 shows the photograph of the NUANS BL2. The left hand side of the photograph is the proton beamline and the center is the target shield. Currently, the accelerator is being adjusted, and the proton beam intensity of 1.5 $\mathrm{mA}$ and $4.2 \mathrm{~kW}$, which are the design values, has not been achieved and a thermal neutron beam measurement is used using a proton beam of approximately $0.1 \mathrm{~mA}$. The neutron intensity used in the measurement is $2 \times 10^{4} \mathrm{n} / \mathrm{s} / \mathrm{cm}^{2}$ at the BL2-short. The assumed thermal neutron energy spectrum is as shown by reference[4]. This accelerator is an electrostatic accelerator and is a DC beam, so neutron energy measurement using TOF measurement is not possible, and the energy spectrum is obtained by calculation. Neutron transmission images are measured with a combination of ${ }^{6} \mathrm{LiF} / \mathrm{ZnS}(\mathrm{Ag})$ Scintillator and cooled CCD. The thickness of the Scintillator is $200 \mu \mathrm{m}$, which is determined by the balance between detection efficiency and spatial resolution. There is one mirror between the CCD and the scintillator to shield the $\gamma$ rays, and the light emission image of the scintillator is measured by the CCD image reflected by the mirror. The cooled CCD is BU53LN from BITRAN Corporation [9]. This CCD has 4008x2672 pixel elements of $9 \times 9 \mu \mathrm{m}$, and can acquire images in 16 bits. Among CCDs in the same price range, this CCD camera is characterized by low noise due to the cooling function by the Peltier element, and the element area is large. This CCD camera is coupled to an $85 \mathrm{~mm}$ F1.4 lens (Nikon Corp.). The lens aperture is usually set to the maximum, and the distance between the camera and the scintillator is changed to adjust the focus.

Figure 2 is a neutron transmission images of an ASTM neutron radiographic sensitivity indicator measured on the 
BL2-short, which L/D value is about 5. The exposure time is twenty minutes. In the image on the left, the gaps created by spacers of about $100 \mu \mathrm{m}$ can be measured as lines. In the right figure, the defocus image due to beam divergence and sample thickness can be seen. In the right figure, two black lines are seen, the right line is clear but the left line is blurred. This is due to the beam divergence and sample thickness, because the $\mathrm{Cd}$ bars are placed on the front and back of the indicator

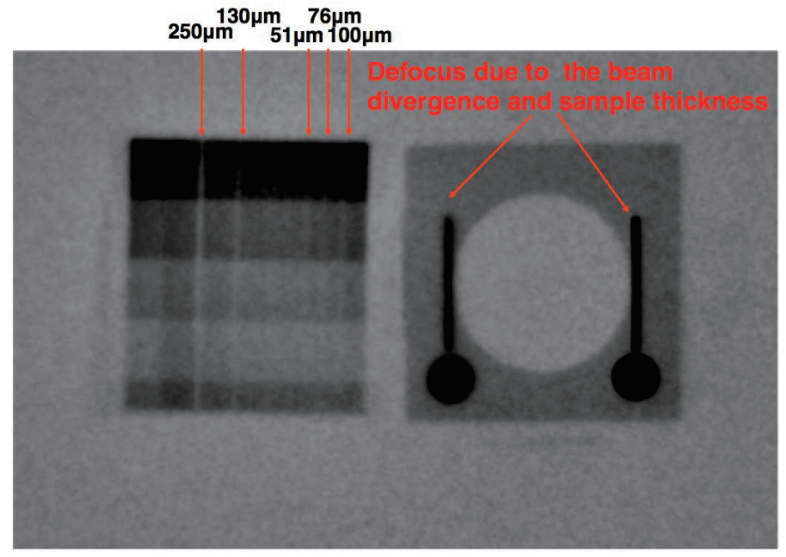

Figure 2. Neutron transmission Image of the ASTM neutron indicator

\section{Focus Adjustment}

In parallel with the construction of the neutron source, we are proceeding with a quantitative evaluation of acquired images. The focus adjustment of the neutron camera used for measurement is usually done with the naked eye. The focus position is depending on the person who works and the measurement conditions. To obtain the same result irrespective on the person who performed it, it was decided to evaluate the focus adjustment quantitatively. For focus adjustment, it is necessary to perform edge extraction on the acquired image. Therefore, the image obtained by the CCD camera is subjected to a noise filter for removing the CCD element error and an FFT (Fast Fourier transform) filter for removing the neutron intensity distribution dependency, and then the second derivative of the space (Laplace calculation ). Since the Laplacian filter is easily affected by noise, it is necessary to eliminate noise such as dot filtering using a noise filter.

Figures 3 shows the images before (upper) and after (lower) the Laplacian filter is applied. The upper figure shows the result of applying the noise filter and bandpass filter to CCD output. The lower figure shows the image obtained by extracting an edge by the Laplacian filter. This sample neutron transmission image was taken at the E2 port of the KUR research reactor.

Figure 4 plots the calculation of the variance of the Laplacian with changing the camera position. This variance value is predicted to take the minimum value when focused. In this figure these values have a minimum at the position is $42.5 \mathrm{~mm}$. This camera position is the same as
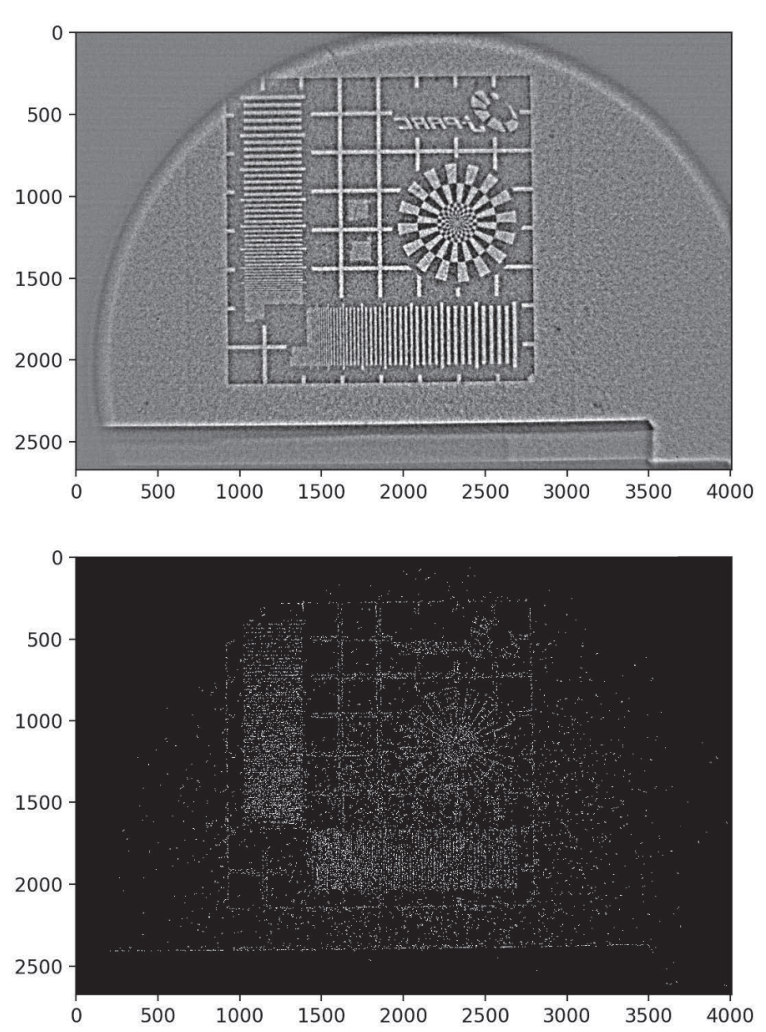

Figure 3. Images before (upper) and after (lower) the Laplacian filter is applied.

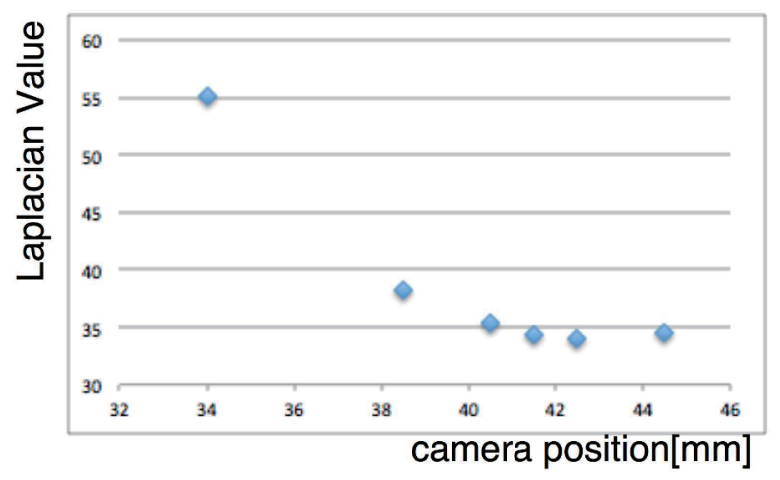

Figure 4. Position dependence of the Laplacian value

the focus point checked by our naked eyes. It may be a method that can be used to adjust the CCD camera's focus in CANS, although this method needs a little more detailed confirmation.

\section{Summary}

The spallation large accelerator facility is versatile and is widely used for precision measurement for academic purposes. On the other hand, a small neutron source using a small accelerator is expected to be used as a familiar neutron source in the future as a device specialized for a specific purpose. Under such circumstances, an accelerator neutron source using low-energy protons with $\mathrm{Ep}=2.8$ 
$\mathrm{MeV}$ was constructed. BL2 is designed as a multi-purpose neutron beamline, and the thermal neutrons generated by the $\mathrm{Be}(\mathrm{p}, \mathrm{n})$ reaction and thermal moderator. Currently being adjusted the accelerator, $2 \times 10^{4} \mathrm{n} / \mathrm{s} / \mathrm{cm}^{2}$ at the BL2short port position neutrons are available. The length from the moderator surface to BL2-short is $50 \mathrm{~cm}$ and the corresponding $\mathrm{L} / \mathrm{D}$ value is 5 . The neutron intensity will be increased in the future.

As part of the quantitative evaluation of neutron measurement, the focus adjustment during image acquisition is digitized. By performing a Laplacian calculation on the image, the edge of the acquired image was extracted and used for focus evaluation.

\section{References}

[1] I.S. Anderson, C. Andreani, J. M. Carpentaer, G. Festa, G. Gorini, C. K. Loong and R. Senesi. Phys. Rep. 654 (2016) 1-58

[2] Katsuya Hirota, PoS(KMI2017)(2017)025

[3] Ikuya Ito, D. Furusawa, K. Hirota, G. Ichikawa, Y. Iwashita, M. Kitaguchi, Y. Kiyanagi, Y. Menjo, Y. Miwata, H. M. Shimizu, K. Tsuchida, Y. Tsuchikawa, Y.
Tsurita, A. Uritani, K. Watanabe, Y. Yamagata, A. Yamazaki, S. Yoshihashi,PoS(KMI2017)68

[4] Y. Tsuchikawa, K. Abo, D. Furuzawa, K. Hirota, G. Ichikawa, S. Imajo, I. Ito, Y. Iwashita, M. Kitaguchi, Y. Kiyanagi, Y. Miwata, K. Saito, H. M. Shimizu, H. Takehista, K. Tsuchida, Y. Tsurita, A. Uritani, K. Watabnabe, Y. Yamagata, A. yamazaki, S. Yoshihashi, JPS Conf. Proc. 22, 011024(2018)

[5] M.R.Hawkesworth, Atomic Energy Review 15, No.2 (1977) 169-220

[6] Yutaka Yamagata, Katsuya Hirota, Jungmyoung Ju, Sheng Wang, Shin-ya Morita, Jun-ichi Kato, Yoshie Otake, Atsushi Taketani, Yoshichika Seki, Masako Yamada, Hideo Ota, Unico Bautista, Oinngan Jia. J Rad. Nucl. Chem. (2015) 305: 787-794

[7] S. Wang, Y. Otake, Y. Yamagata, T. Nagae, H. Fujioka, M. Hirose, Y. Kiyanagi, M. Furusaka, K. Hirota, Physics Procedia 60 (2014) 319

[8] S. Tasaki, T. Nagae, M. Hirose, Y. Yamashita, K. Hironaka, Y. Abe, Y. Yamagata, Y. Otake, K. Hirota, Physics Procedia 60 (2014) 181

[9] https://www.bitran.co.jp 